\title{
Simulation of the Robot Based Machining Process for Prosthetic Socket Positive Mold
}

\author{
Xiangyi $\mathrm{Li}^{1,2, a}$, Lifeng Guo $2, \mathrm{~b},{ }^{*}$ \\ ${ }^{1}$ School of Mechanical Engineering, Beijing University of Chemical Technology, Beijing, China \\ ${ }^{2}$ School of Mechanical Engineering, Beijing Institute of Petrochemical Technology, Beijing, China \\ alixiangyi763@163.com, bguolifeng@bipt.edu.cn \\ *Lifeng Guo
}

Keywords: industrial robot; prosthetic socket; 3D scan technology and modeling; path planning; machining process simulation

\begin{abstract}
Based on KUKA robot processing platform and Robotmaster software, the machining process simulation technology of prosthetic socket positive mold was studied. The KUKA KR210 control system KR C4 is extended with PROFIBUS/DP communication to integrate the machining spindles for industrial robots with machining capabilities. Based on this platform, a simulation platform of robot machining process is established. The three-dimensional model of positive model was established by optical three-dimensional scanning method. In view of the positive mold surface features, the machining process planning was completed in Mastercam. Robotmaster software is used to realize offline programming and simulation of robot processing. The realization of robot machining process simulation laid a good foundation for physical processing of prosthetic socket.
\end{abstract}

\section{基于工业机器人的假肢接受腔阳模加工过程仿真 李向义 ${ }^{1,2, a}$, 郭丽峰 $2, b,{ }^{*}$ \\ 1 北京化工大学 机电工程学院, 北京, 中国 \\ 2 北京石油化工学院 机械工程学院, 北京, 中国 \\ alixiangyi763@163.com, bguolifeng@bipt.edu.cn \\ *郭丽峰}

关键词: 工业机器人; 假肢接受腔; 三维扫描及建模; 路径规划; 加工仿真

摘要: 基于KUKA机器人加工平台和Robotmaster软件, 研究了假肢接受腔阳模加工过程仿真 技术。应用PROFIBUS/DP通讯对KUKA KR210控制系统KR C4进行扩展, 集成加工主轴, 使 工业机器人具有加工能力, 以此为基础, 建立机器人加工过程仿真平台。通过光学三维扫描 方法建立假肢接受腔阳模计算机三维模型。针对接受腔阳模曲面特征, 在Mastercam中完成加 工工艺规划。应用Robotmaster软件实现机器人加工过程的离线编程、仿真。机器人加工过程 仿真的实现为假肢接受腔实物加工打下了良好基础。

\section{1. 引言}

目前在临床上，假肢接受腔阳模的加工多采用石膏绷带取型和手工修型的传统制作方法， 该方法工艺步骤繁琐，费时费力[1]，其质量很大程度上取决于假肢技师的修模技术。

上世纪中后期，一些学者开始将CAD/CAM技术应用于假肢设计、制作领域[2]，经过几 十年发展已有一些解决方案推向市场, 典型代表有法国rodin4D公司推出的机器人雕刻系统 
[3], 和拿大VORUM公司的七轴机器人假肢雕刻系统[4]等。这些产品将假肢CAD技术与机器 人加工CAM技术相结合,使假肢接受腔的制作实现了信息化、精度化、高效化,但售价昂贵， 难以推广。王喜太[5]、郭宏[6]等分别对假肢接受腔计算机辅助设计与制造系统、个性化假肢 快速成型系统进行了研究, 与传统法手工制作假肢接受腔相比, 这些系统有了很大进步, 但 相关技术还有待完善和成熟，短时间内难以形成统一标准，距商业化应用还需较长时间。

随着机器人技术的快速发展, 一些专用于机器人加工的离线编程仿真软件的应用日益广 泛, 如RobotMaster、PowerMill、RobotWorks、SprutCAM等[7], 这为基于机器人的加工过程 规划和离线仿真提供了便利条件。本文基于KUKA机器人加工平台和Robotmaster软件, 建立 虚拟仿真环境，对假肢接受腔阳模加工工艺进行了分析和过程仿真。

\section{2. 机器人加工系统建模}

基于工业机器人假肢接受腔阳模加工过程仿真技术流程主要包括接受腔阳模三维建模, Masercam中加工参数设定及刀具路径规划, Robotmaster中仿真参数设定, 机器人加工仿真等 几个步骤，如图1所示。

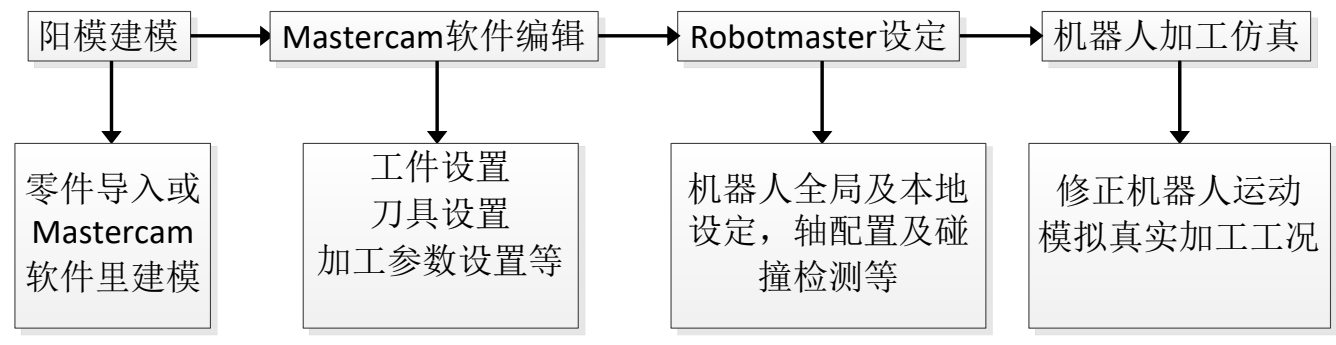

图1 技术方案流程图

机器人加工系统由上位PC机, 机器人本体及其控制系统, 加工主轴及其控制器 3 大部分 组成 $[8]$ 。上位PC机负责接受腔阳模的三维建模, 工艺规划及轨迹的生成。选用KUKA KR210 型六自由度工业机器人, 搭载KR C4控制柜, 外部控制系统通过Ethernet和Profibus/DP等方式 实现和此控制柜的通讯连接。对机器人加工系统建立三维模型, 如图2中(a)、(b)所示。

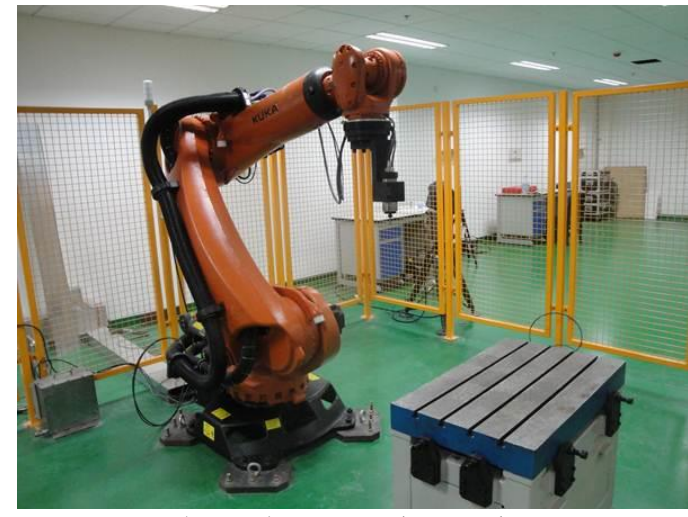

(a) 加工机器人实物系统

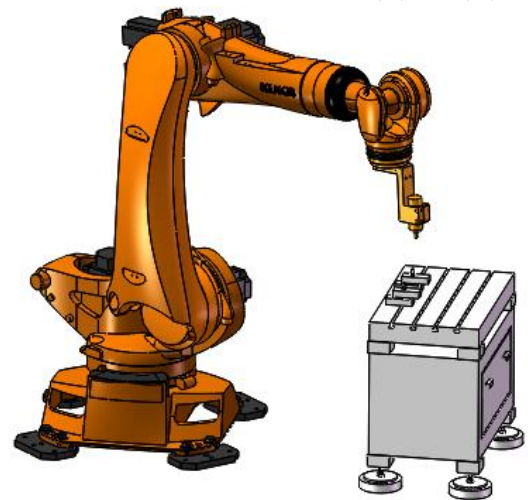

(b) 加工机器人三维建模

图2 机器人加工系统

\section{3. 接受腔阳模建模}

使用三维扫描仪对假肢接受腔阳模进行扫描, 再借助相应软件对三维扫描数据进行处理 进而实现三维建模。扫描系统由Artec Eva三维扫描仪、电动转台、计算机, 以及Artec Studio 图形处理软件等部分组成, 如图3所示。 


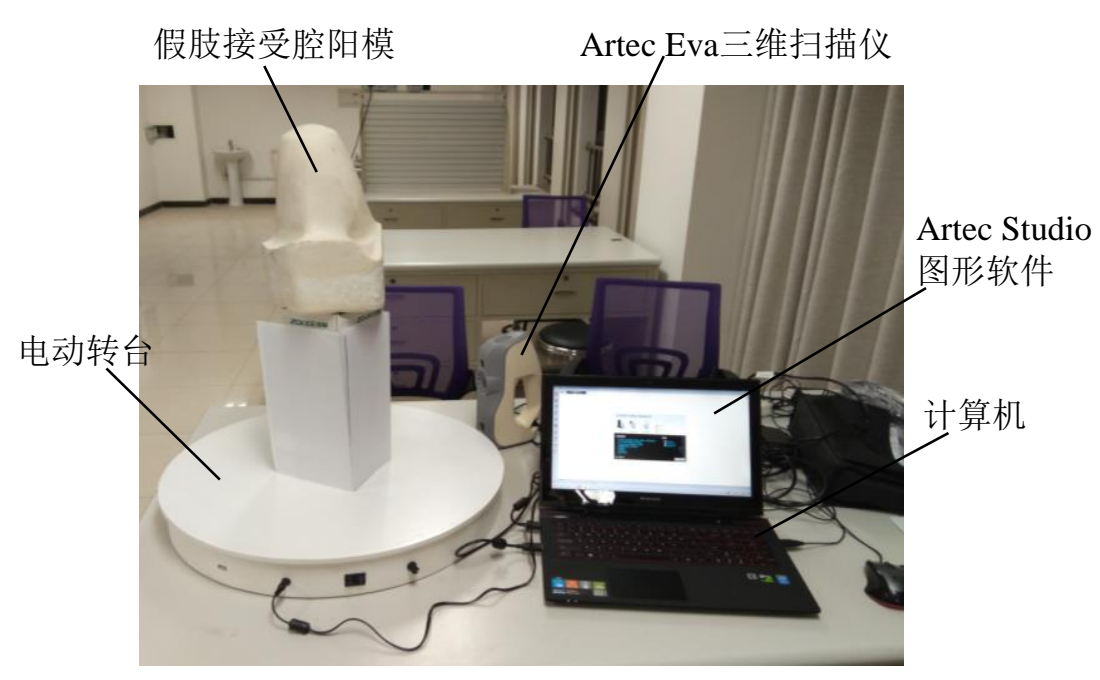

图3 接受腔阳模扫描系统

扫描得到的原始测量数据经“配准”、“边缘处理”、“纹理处理”、“合成”、“文件输出”等处 理, 生成接受腔阳模三维模型, 如图4所示。

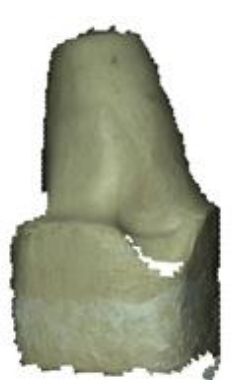

(1) 扫描

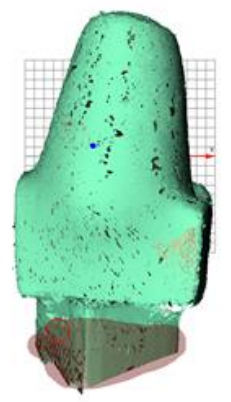

(2) 擦除

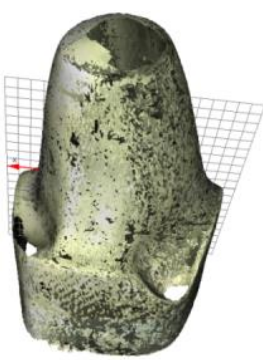

(3) 补洞

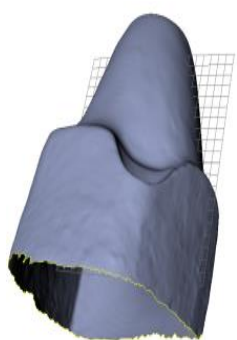

(4) 边缘处理

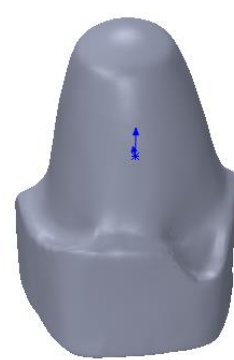

(5) 实体合成

图4 由Artec网格文件到solidworks实体文件

\section{4. 加工路径规划}

假肢接受腔阳模外形几何尺寸为 $194 \mathrm{~mm} （$ 长 $) \times 176 \mathrm{~mm} （$ 宽）×286mm（高），是一个复 杂的不规则异型曲面。由图5可知, 接受腔阳模的顶部和底部曲面曲率变化小, 刀具在加工过 程中轴向方向过渡平缓, 而口型圈部分曲面曲率变化较大, 刀具轴的方向在这部分变化急剧。

口型圈以上的部分和以下的部分设置刀具轴的方向为加工Z轴的法线方向, 口型圈部分设 置为沿曲面的法线方向。由于法线方向无法满足整个口型圈部分曲面的加工, 因此设置刀具 沿加工方向的引线角度和侧边倾斜角度来调节。为得到接受腔阳模表面所需粗䊁度精度等级, 采用先粗加工, 后精加工, 最后清角加工的加工工序。

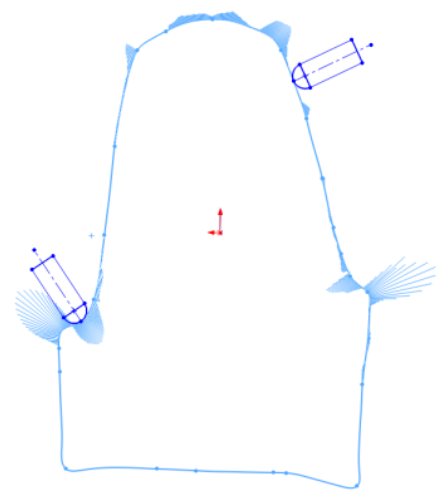

图5＼cjkstart接受腔阳模曲面曲率及加工刀具姿态 
在Mastercam中输入接受腔阳模IGS文件, 机床类型选择铣床。粗加工以曲面挖槽加工方 式进行层削, 精加工采用曲面五轴加工平行切削方式, 清角加工采用等高外形铣削。坏料的 尺寸选用 $210 \mathrm{~mm} \times 190 \mathrm{~mm} \times 300 \mathrm{~mm}$ 。选择刀具直径分别为 $8.0 \mathrm{~mm}$ 圆鼻刀和 $6.0 \mathrm{~mm}$ 的球刀作为接 受腔阳模粗加工和精加工的刀具。粗加工步进量设为 $2 \mathrm{~mm}$, 加工余量设置为 $1 \mathrm{~mm}$ 。精加工步 进量设为 $0.8 \mathrm{~mm}$, 加工余量设置为 $0.3 \mathrm{~mm}$ 。清角加工选用直径为 $4 \mathrm{~mm}$ 的圆鼻刀。在刀具路径 规划中, 以曲面挖槽加工方式自上往下进行层削, 进给速率、下刀速率、提刀速率、主轴转 速分别设置为 $400 \mathrm{r} / \mathrm{min} 、 500 \mathrm{r} / \mathrm{min} 、 1200 \mathrm{r} / \mathrm{min} 、 1600 \mathrm{r} / \mathrm{min}$ 。以坏料顶面的一个棱角顶点为对 刀点, 刀具安全高度设置为 $50 \mathrm{~mm}$, 切削方式设置为双向。粗加工、精加工、清角加工过程如 图6所示。

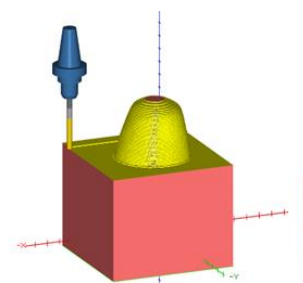

(1)

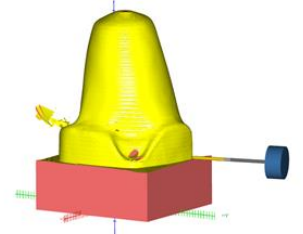

(3)

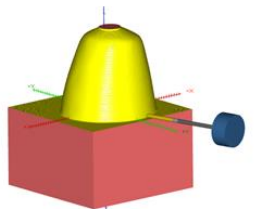

(2)

(1) 粗加工

(2) 精加工

(3) 清角加工

图6 接受腔阳模不同加工阶段

每个加工阶段设置完刀具及刀柄后, 都要选取接受腔阳模曲面的待加工部分, 设置切削 方式、刀具轴向控制、碰撞控制及共同参数。以精加工阶段为例, 接受腔阳模的刀具路径规 划完成后, 如下图7所示。

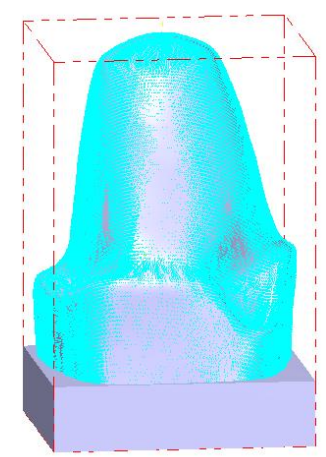

图7 Mastercam中接受腔阳模加工路径

\section{5. 加工过程仿真}

接受腔阳模的刀具路径规划好之后，启动Robotmaster，在“刀具操作管理器”中将铣削机 床设置为KUKA机器人, 对其进行全局坐标系和本地坐标系设置, 将接受腔阳模的CNC机床 刀具路径配置成机器人能用的刀具加工路径。

机器人全局坐标系主要对机器人及加工主轴类型、接受腔阳模坐标数据, 机器人起始点、 结束点等进行设置。机器人本地坐标系主要对轴配置的工具补差方式, 旋转方式、角度及旋 转增量进行设置。机器人仿真栏中主要对夹具和STL公差进行设置。

考虑到接受腔阳模的高度和机器人位姿摆动, 为方便加工, 接受腔阳模的基坐标数据的 X、Y、Z栏分别设置为 $1800 \mathrm{~mm} 、 200 \mathrm{~mm} 、 200 \mathrm{~mm}$, 机器人起始点和结束点位置角度设置为 
(90，-45，-45,0，-95,0)，球面补差方式, 默认Z轴不旋转, 旋转增量设置为 5, STL公差 0.02 。 机器人对接受腔阳模的加工过程仿真, 如图8所示, 加工仿真过程表明, 机器人加工系统不会 发生碰撞和干涉。

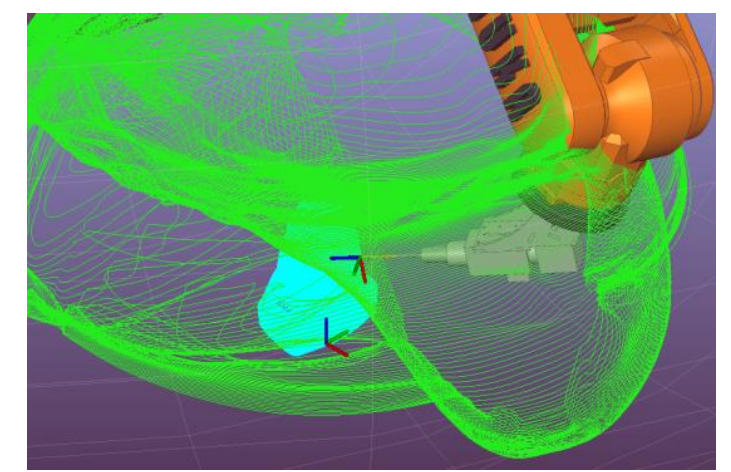

图8 Robotmaster中接受腔阳模机器人加工过程仿真

\section{6.结束语}

基于KUKA机器人加工平台，在Masercam和Robotmaster中实现了假肢接受腔阳模加工过 程的仿真。机器人加工过程仿真的实现从理论上确定了加工机器人运动路径及加工参数, 为 假肢接受腔阳模的实物加工打下良好基础, 也为接受腔阳模的自动化加工提供了一种可行的 技术方案。

\section{致谢}

本文为北京市教育委员会市属高校创新能力提升计划项目“大型医学影像设备SPECT机 电系统研制” (编号: TJSHG201310017035) 和北京市属高等学校青年拔尖人才培育计划项目 “基于工业机器人的假肢接受腔柔性加工系统研究”（编号：CIT\&TCD201404104）的阶段性 成果之一。

\section{References}

[1] Guo Xin, Prosthtic socket computer aided manufacturing system, Hebei Univesity of Technology, pp. 1-50, 2002.

[2] Cao Xuejun, NI Xiansheng, LIU Wenyong, et al. Geometric modeling of prosthetic socket, Chinese Journal of Rehabilitation Theory and Practise, vol.15, pp. 283-285, 2009.

[3] ROBOT VICTOR 7-axis machining robot, RODIN SAS. http://rodin4d.com/en/Products /manufacturing.

[4] Robot 7-Axis Carving Machine, Vorum Research Corp. http://vorum.com/cad-cam-prostheticorthotic/carvers/.

[5] Wang Xitai,Yan Heping,Yang Peng et al.Prosthetic socket computer aided design and menufacturing system, Chinese Journal of Rehabilitation Medicine, vol.17, pp. 284-286, 2002.

[6] Guo Hong, Design and research of rapid babrication system of prosthetic socket, Liaoning Technical University, pp. 1-58, 2004.

[7] Wei Zhili, Song Zhiguang, Guo Ruijin, Review on commercial systems for off-line programming of robot, Electrical Automation, vol. 54, pp. 180-183, 2016. 
[8] Guo Lifeng, Zhao Xiaofei, Zhou Canfeng, et al. Research on flexible manufacture system based on kuka industrial robot, Journal of Beijing Institute of Petro-chemical Technology, vol. 32, pp. 45-49, 2015. 